\title{
Was sind Online-Inhalte wert?: Determinanten der Kaufentscheidung für redaktionelle Paid Content-Produkte
}

Lischka, Juliane A ; Hutter, T ; Rademacher, Patrick

\begin{abstract}
Trotz des wieder erstarkten Interesses von Medienunternehmen am Thema Paid Content spielen Paid Content-Erlöse oft noch eine untergeordnete Rolle. Welche Determinanten die Kaufentscheidung bei unterschiedlichen Zielgruppen beeinflussen, ist sowohl der Medienpraxis als auch der Wissenschaft häufig noch unklar. In der vorliegenden Untersuchung werden die Ergebnisse einer Online-Befragung von 2.850 Schweizer Mediennutzern zu ihren Kaufentscheidungsfaktoren und Nutzungsgewohnheiten von Paid Content dargestellt. Vor allem der kostenpflichtige Inhalt selbst, sein Preis und das Zahlungssystem determinieren die Kaufentscheidung. Die jeweiligen Informationsbedürfnisse Paid Content-affiner Nutzergruppen (v. a. Hintergrundinformationen und Reportagen, Archive und Datenbanken sowie aktuelle Informationen) können von Medienunternehmen vergleichsweise einfach befriedigt werden.
\end{abstract}

Posted at the Zurich Open Repository and Archive, University of Zurich

ZORA URL: https://doi.org/10.5167/uzh-96370

Journal Article

Originally published at:

Lischka, Juliane A; Hutter, T; Rademacher, Patrick (2012). Was sind Online-Inhalte wert?: Determinanten der Kaufentscheidung für redaktionelle Paid Content-Produkte. Medienwirtschaft, 9(4):12-23. 


\section{Was sind Online-Inhalte wert? Determinanten der Kaufentscheidung für redaktionelle Paid Content-Produkte}

Trotz des wieder erstarkten Interesses von Medienunternehmen am Thema Paid Content spielen Paid Content-Erlöse oft noch eine untergeordnete Rolle. Welche Determinanten die Kaufentscheidung bei unterschiedlichen Zielgruppen beeinflussen, ist sowohl der $\mathrm{Me}$ dienpraxis als auch der Wissenschaft häufig noch unklar. In der vorliegenden Untersuchung werden die Ergebnisse einer OnlineBefragung von 2.850 Schweizer Mediennutzern zu ihren Kaufentscheidungsfaktoren und Nutzungsgewohnheiten von Paid Conten dargestellt. Vor allem der kostenpflichtige Inhalt selbst, sein Preis und das Zahlungssystem determinieren die Kaufentscheidung Die jeweiligen Informationsbedürfnisse Paid Content-affiner Nutzergruppen (v. a. Hintergrundinformationen und Reportagen, Archive und Datenbanken sowie aktuelle Informationen) können von Medienunternehmen vergleichsweise einfach befriedigt werden.

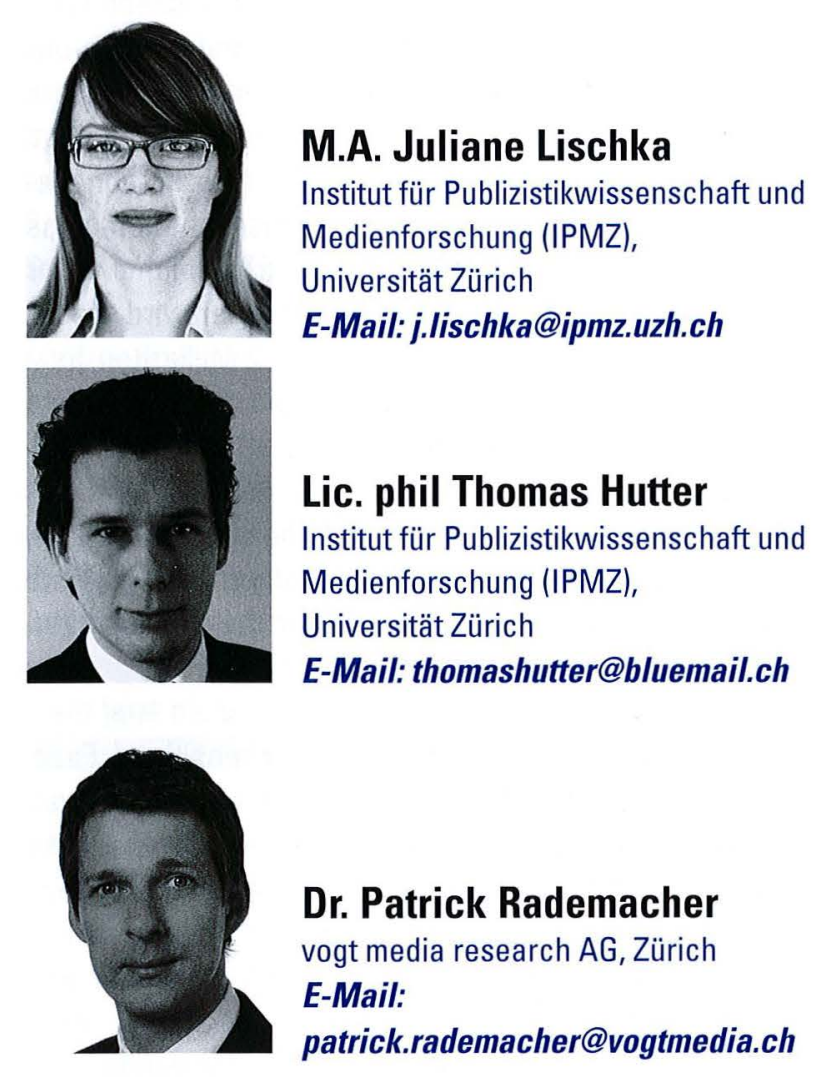

Schlüsselbegriffe: Paid Content I Paywall | Kaufentscheidungsfaktoren I Conjoint Analyse

\section{Kernthesen:}

- Obwohl die allgemeine Zahlungsbereitschaft für Online-Angebote gering ist, kann für spezifische Inhalte ein Preis verlangt werden.

- Der kostenpflichtige Inhalt selbst, sein Preis und das Zahlungssystem determinieren die Kaufentscheidung.

- Für Hintergrundinformationen, Archive und Datenbanken besteht ein hohes Paid Content-Potenzial.

\section{Einleitung}

Für Medienunternehmen, allen voran Verlage, ist es nach wie vor eine Herausforderung, mit Online-Inhalten Nutzererlosse zu erzielen. Paid Content-Strategien müsse tierphase hinaus weiterentwickelt werden. Rückläufige tierphase hinaus weiterentwickelt werden. Rückläufig Werbeerlöse für klassische Printmedien verstärken dabei die Notwendigkeit für Verlage, Erlöse zum einen von der Konsumentenseite und zum anderen uber den Vertriebskanal Online zu generieren, und zwar sowohl uber das stabei der Implementierung von Paid Content ist es, dass die für welche Inhalte zahlungsbereit sind. Unter Paid ContentProdukten verstehen wir kostenpflichtige, redaktionell erstellte Inhalte, die online angeboten werden.

Der Beitrag ist wie folgt strukturiert: Zunächst analysieren wir den Status Quo von Paid Content in einer von Free Content geprägten Konkurrenzsituation und beschreiben Kaufentscheidungskriterien für Paid Content-Angebote. Anschließend werten wir die Ergebnisse einer Online-Befragung von 2850 Schweizer Online-Mediennutzern fragung von 2.850 Scheizer Online-Mediennutzern aus. Dabei stellen wir Charakteristika von Paid Content-Nutzern und bevorzuge joint Analyse dar. Weiter analysieren wir Nutzercluster und evaluieren das Potenzial an Paid Content fur diese Cluster Abschließend diskutieren wir die Ubentragbarkeit der Ergebnisse auf den deutschen Markt und leiten Schlussfogerungen für Medienmanager ab.

1 Status quo der Etablierung von Paid Content

Derzeit spielen Paid Content-Erlöse für Verlage oft noch eine untergeordnete Rolle. Aufgrund der unsicheren Aussichten zögern viele Verlage nach wie vor, Paid Content tatsächlich anzubieten. Und bei den Unternehmen, die den Schritt Richtung Paid Content bereits gewagt haben, können die Online-Erlöse die wegbrechenden Einnahmen aus dem strukturell rückläufigen Printgeschäft bei weitem nicht aufwiegen (Rosenstiel et al. 2012). Bei der Umstellung von kostenfreien auf kostenpflichtige Angebote müssen Medienunternehmen zudem damit rechnen, dass ihre bisherigen User zu anderen Websites wechseln, auf denen herigen User zu anderen Websites wechseln, auf denen ahnliche Inhate frei zugangnglich sind (Pauwels/Weiss 2008) Zu beobach is die Anzahl der Ontes nach der Einfuhrung einer Paywall rückgegangen (BBC 2010). Mit weniger Traffic werden Websites für die Werbewirtschaft jedoch auch weniger
attraktiv.

Ein Preis signalisiert zudem Qualität zu ungunsten der kostenfreien Angebote.

Die Einführung einer Paywall bedeutet allerdings nicht zwangsläufig einen signifikanten Rückgang der Nutzerschaft: Bei der New York Times ließen sich nach Einführung einer "weichen" Bezahlschranke in Form einer Metered Paywall im März 201120 Artikel kostenlos abrufen. Seit März 2012 wurde dies auf zehn Artikel reduziert, was laut Unternehmensangaben innerhalb von drei Monaten sogar zu einem Abonnentenzuwachs von $13 \%$ führte (The New York Times Company 2012). Im Okn New York Times Company 2012). Im Oktober 2012 hat nu auch die Schweizer Tageszeitung Neue Zürcher Zeitung NZZ) eine Metered Paywall nach dem Vorbild der New laubt das kostenlo Abrufen von 20 Artikeln pro Monat.
Dadurch erhofft sich die NZZ, Online-Nutzererlöse zu erzielen, ohne einen spürbaren Rückgang ihrer Reichweite verschmerzen zu mussen (Hogenkamp et al. 2011).' Mit einem Online-Abonnement oder zumindest einer Registrierung können Verlage wie etwa die NZZ Informationen über die Nutzerschaft sammeln, die relevant für Werbetreibende sind, und dadurch ggf. ihre Werbeerlöse erhöhen.

Gerade auch die Etablierung des mobilen Internets anhand von Smartphones und Tablet-PCs lässt das längere Zeit totgesagte Thema Paid Content wieder erstarken (Chyi 2012; Hall 2010; Malone 2012; Mitchell et al. 2012). Die Verbreitung von Smartphones in der Schweiz hat von 2010 bis 2012 von 29 \% auf etwa 50 \% der Bevölkerung zugenommen (NET-Metrix AG) und liegt damit etwa gleichauf mit den USA (Mitchell et al. 2012). In Deutschland ist die Smartphone-Verbreitung mit etwa $29 \%$ der Bevölkerung zwar aktuell noch geringer (Google 2012), insgesamt verbessert sich die technische Ausstattung der Bevölkerung jedoch rasant, und damit wächst auch die potenzielle Nutzerschaft von mobilem Paid Content. So besitzen inzwischen vier von zehn Mobiltelefonnutzern in Deutschland ein Smartphone (BITKOM 2012). Laut einer aktuellen Umfrage in den USA führen mobile Geräte dazu, dass mehr Nachrichten konsumiert werden und eine Multi-Plattform-Nutzung entsteh (Mitchell et al. 2012). Mobiler Paid Content wird als Möglichkeit gesehen, die Rezipienten gleich zu Beginn der Entwicklung an kostenpflichtige Angebote zu gewöhnen. So sind beispielsweise die Inhalte der Apps der Schweizer Boulevardzeitung Blick oder der deutschen Boulevardzeitung BILD nur kostenpflichtig beziehbar.

2 Kaufentscheidungskriterien für Paid Content

Grundsätzlich können Kaufentscheidungen für oder gegen ein Produkt auf Einflussfaktoren verschiedener Ebenen zurückgeführt werden. Ein Produkt erfüllt sowohl einen funktionalen als auch einen psychologischen Nutzen (Paul et al. 2009). Entsprechend lassen sich funktionale und psychologische Kaufentscheidungskriterien unterscheiden

Für e-commerce-Angebote spielen auf der funktionalen Ebene neben der Exklusivität des Inhalts ein einfacher Zugang und schnelle Zahlungsabwicklung eine wichtige Rolle (Wang et al. 2005). So verringert sich die Kaufattraktivität bei einem zu komplexen Bezahlungssystem (Rademacher 2007) oder bei einer qualitativ schlechten Website (Hangjung Zo/Ramamurthy 2009). Für den funktionalen Nutzen von Paid Content ist u. a. der Inhalt selbst ausschlaggebend. Dabei ist Inhalt nicht gleich Inhalt: Aus Sicht von Branchenexperten sind gerade allgemeine Nachrichten nicht dazu geeignet, kostenpflichtig angeboten zu werden (Herbert/Thurman 2007) da ihr Inhalt substituierbar ist Dies ist eispielswe

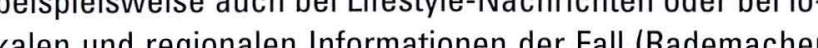
2007: 77).

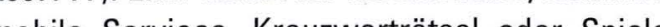


gefordert werden (Herbert/Thurman 2007). Zeitelastische Informationen wie Verkehr und Wetter oder auch weni zeitelastische Dienstleistungen mit Servicecharakter wie Haushaltstipps liefern direkte Handlungsempfehlungen
und somit einen Zusatznutzen, für den ein Preis verlangt werden kann (Rademacher 2007: 77). Servicecharakter kann auch durch Bündelung von Inhalten erreicht werden wodurch Online-Angebote insgesamt von einer höheren Nutzerloyalität profitieren können (Zheng ot at. 2012).

Ein psychologisches Entscheidungskriterium, das die Kaufatraktivität von Online-Inhalten steigett, bese den bereits gemachen jeweiligen Medium oder mit ener Prinvesion gute Efahiunge chen konte ist en zu erwerber (R. Varian (Radenacher 2007). Weilere psychologische hörige Oualito Markenwahrnehmung sowie die dazugeduktnutitatsourteilung formen den individuellen Produktnutzen mit. Gerade die Reputation der Medienmarke kann über den funktionalen Nutzen hinaus symbolischen Nutzen stiften (Siegert 2001). Markenbekanntheit beeinflusst den wahrgenommenen Wert des Produkts und die Zahlungsbereitschaft des Konsumenten positiv (Dou 2004 350). Ein Preis signalisiert zudem Qualität zu ungunsten de kostenfreien Angebote (Pauwels/Weiss 2008). Gerade be Erfahrungs- oder Vertrauensgütern wie Nachrichten fältt es dem potenziellen Konsumenten schwer, den subjektiven Nutzen und die Qualität eines Inhalts im Voraus abzuwägen. Das Vertrauen, das Kunden in eine Marke haben kann ausschlaggebender für die Kaufentscheidung sein als der Preis (Kim et al. 2012) In Verbindung mit dem OulitätssigPreis (Kim et al. 2012). In Verbindung mit dem Qualitätssignal eires Preises neh dienmarke² unter bestinn

Internetnutzer sind also aufgrund von Free Content und dem fehlenden sub jektiven Mehrwert von Paid ContentAngeboten unwillig, für publizistische Online-Angebote zu bezahlen.

Einen Preis auf digitalen Märkten durchzusetzen, ist jedoc schwerer als auf „klassischen" Märkten, da sich Inhalte auf digitalen Märkten durch ihre einfache Verfügbarkeit sowie durch niedrige Transaktionskosten auszeichnen (Stahl 2005: 31), zumal die gleichen Medienunternehmen einen Preis für etwas verlangen, was sie bisher kostenfrei ang Pren hall stößt jedoch dann auf ancono han etwas hohe Zahlungsberelschat, wenn die Zahlung als Notwendigleit herausgestellt wird anstatt als zusätzlicher Profit, wie eine aktuelle Untersuchung am Beispiel
New York Times-Paywall zeigt (Cook/Shahzeen 2012).
Auf digitalen Märkten können Rezipienten die Angebote einfacher vergleichen, was das Preisniveau negativ beeinflusst. Das Vorgehen, gesuchte Informationen gratis zu "googeln , sei noch stark verbreitet unter den Internetnutzern (Wan et al. 2005). So lässt die Gratismentalität die Zahlungsbereitschaft deutlich sinken, wie Dou (2004) in einer OnlineBefragung für den amerikanischen Markt nachgewiesen hat In einer aktuellen Online-Befragung amerikanischer OnlineNutzer (Chyi 2012) ist die Zahlungsbereitschaft für Paid Content nach wie vor sche tent Tagent Paywall oder ei verien die Befragten für die Printversion 8 Dollar, für die Web-Version gut 3 Dollar und für ein App 1,5 Dollar im Monat zahlen. Die wark Con der

Andererseits können die Opportunitätskosten für die Suche nach alternativen (Gratis-) Informationsquellen für zeitelastische „Fast Moving Information Goods" schlichtweg z hoch sein, so dass die Nutzer auf eine Free Content-Suche verzichten. Entsprechend erhöht Dringlichkeit die Kaufattraktivität von journalistischen Inhalten (Rademacher 2007). Ei weiterer positiver Prädiktor für die Zahlungsbereitschaft is das Interesse an bestimmten Nachrichten (Chyi 2012). Ums wicktiger ist es, dass Paid Content für den Nutzer wertvoll, einzigartig und nicht anderweitig kostenlos zugänglich is (Fredrich 2005; Rademacher 2007). So waren gemäß einer BITKOM-Untersuchung aus dem Jahr 2010 prinzipiell knap $40 \%$ der Deutschen net zu bezahlon falls dieser nirgends mehr gratis ang Interwürd I In und und dem fehlenden subjektiven Mehrwert von Paid ContentAngeboten unwillig, für publizistische Online-Angebote zu

\section{Studiendesign und Analyse}

Bisher durchgeführte Studien zum Thema Paid Content beschäftigen sich vor allem damit, die Zahlungsbereitschaft der Konsumenten für kostenpflichtige Angebote zu eruieren. Dies geschieht meistens mittels einer Bestandsaufnahme des Paid Content-Marktes (Oelbermann/Reibnitz 2003; Breunig 2005 The Nielsen Company 2010), einer empirischen Nutzerbefragung, um deren Zahlungsbereitschaft aufzuzeigen (Buchholz et al. 2004; Fredrich 2005), oder mittels einer Analyse der kostenpflichtigen Angebote (Dou 2004; Nitschke/Sattler 2005; Rademacher 2007). Dabei beschränkt sich die Paid Content-Forschung auf existierende Produkte. Ein Produkt kann jedoch nicht nur als Gesamtpaket sondern auch als ein Bündel von verschiedenen Eigenscheften verstanden werden. Diese vererchere verence Kaufin Prolug in Prodakeigenschaten kann daher festgestellt werden, ob der potenziele Konsunent ungleiche Praferenzen und unter-
Produkteigenschaften von Paid Content-Angeboten aufweist. Die vorliegende Studie möchte genau hierzu einen Beitrag eisten. Daher werden die folgenden Forschungsfragen gestellt:

- Wer sind die Nutzer von Paid Content?

- Welche Produkteigenschaften und Attribute von Paid Content-Ancuboten führen zur höchsten Kaufwalrscheinlichkeit?

- Welche Rolle spielt der Preis?

Um die Forschungsfragen zu beantworten, wurde eine $0 \mathrm{n}$ line-Befragung durchgeführt. Der Online-Fragebogen wurde von 2.850 Nutzern und Nichtnutzern von Paid Content ausgeüllt und war auf Webseiten von fünf großen, deutschsprachigen Schweizer Online-Medien für vier Wochen im Dezember 2010 geschaltet $^{3}$. Neben Online-Mediennutzung, Paid Content-Erfahrung sowie Demographie beinhaltet der Fragebogen einen Teil mit Fragen, die mittels einer adaptiven Conjoint Analyse (ACA) ausgewertet werden. Dieser Teil dient dazu, diejenigen Produktmerkmale von Paid Content ausfindig zu machen, die den höchsten Einfluss auf die Kaufentscheidung haben. Der Fragebogen orientiert sich an Mögerle (2009) und Fredrich (2005), deren theoretischer und methodischer Auf-

Tab. 1: Soziodemographische Merkmale der Online-Mediennutzer, Paid Content-Nichtnutzer und Paid Content-Nutzer

\begin{tabular}{|c|c|c|c|c|}
\hline \multirow[t]{2}{*}{ Merkmale } & & $\begin{array}{l}\text { Online-Medien- } \\
\text { nutzer }\end{array}$ & $\begin{array}{l}\text { Paid Content- } \\
\text { Nichtnutzer }\end{array}$ & Paid Content-Nutze \\
\hline & $\begin{array}{l}\text { Fallzahl } \\
\text { (Anteil) }\end{array}$ & $\begin{array}{l}n=2.850 \\
(100 \%)\end{array}$ & $\begin{array}{l}n=2.237 \\
(78,5 \%)\end{array}$ & $\begin{array}{l}n=613 \\
(21,5 \%)\end{array}$ \\
\hline \multirow{2}{*}{ Geschlecht } & Weiblich & $21,4 \%$ & $24,1 \% \%^{*}$ & $11,7 \% \%^{*}$ \\
\hline & Männlich & $78,6 \%$ & $75,9 \% \%^{*}$ & $88,3 \%{ }^{*}$ \\
\hline Alter & Median & 40,5 & 40 & 42 \\
\hline \multirow{2}{*}{$\begin{array}{l}\text { Höchster } \\
\text { Bildungsabschluss }\end{array}$} & $\begin{array}{l}\text { Ohne } \\
\text { Hochschulstudium }\end{array}$ & $62,6 \%$ & $66,1 \%{ }^{*}$ & $49,8 \%{ }^{*}$ \\
\hline & $\begin{array}{l}\text { Mit } \\
\text { Hochschulstudium }\end{array}$ & $37,4 \%$ & $33,9 \%{ }^{*}$ & $50,2 \%{ }^{*}$ \\
\hline \multirow{3}{*}{$\begin{array}{l}\text { Persönliches } \\
\text { Einkommen }\end{array}$} & Bis 8.000 CHF (6.600 Euro) & $50,8 \%$ & $53,2 \%{ }^{*}$ & $41,9 \%{ }^{*}$ \\
\hline & $8.000 \mathrm{CHF}$ oder mehr & $23,3 \%$ & $20,2 \%{ }^{*}$ & $34,7 \%{ }^{*}$ \\
\hline & Keine Angaben & $25,9 \%$ & $26,6 \%$ & $23,3 \%$ \\
\hline
\end{tabular}

A/2012 MedienWirtschaft 15 bau eine passende Grundlage für die Operationalisierung de

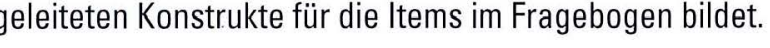

Die Datenanalyse wurde mit Hilfe von Regressionen, ClusterAnalysen und ACA anhand multipler Regressionen durchgezerlegt, die den Befragten einzeln und gebündelt präsentiert werden. Die Befragten beurteilen Produkteigenschaften soAus den Präferenz- sowie Relevanzurteilen der Befragten die Coniont Analyse prädestiniert für Anwendungsgit ist der Preishestimmung, der Produktneugestaltung und der Marktsegmentierung.

Der vorliegende Datensatz wurde auch in einer weiteren Analyse zur Paid Content-Akzeptanz herangezogen (Hutter 12). Darin geht es zusatzlich um Motivationen und Gratifikationen zur Nutzung von Online-Medien. wie Produktbündel hinsichtlich ihrer Präferenz und Relevanz. 

einmal kostenpflichtige, redaktionelle Online-Inhalte gekauft rer demographischen Merkmale (Tabelle 1). gung weiblich. Dieser geringe Anteil steht in Einklang mit der Erkenntnis, dass Männer bei der Nutzung von Online-Medien

\section{Ergebnisse}

4.1 Wer sind die Nutzer von Paid Content?

4.1.1 Nutzerprofil von Online-Mediennutzern und Paid Content-Nutzern

Die Gesamtstichprobe umfasst 2.850 Online-Mediennutzer $(100 \%)$. Von diesen hat gut ein Fünftel bereits mindestens einmal Koraten werden in der Gruppe der Paid Content. Dutzer zefrast Folger zen Folgenden did Gonnutzer

Der typische Online-Mediennutzer ist männlich, Anfang 40 und gut gebildet. Lediglich jede fünfte Befragte ist in dieser Befraübervertreten sind (Mögerle 2009: 261). Der Altersmedian der Online-Mediennutzer liegt leicht unter dem Gesamtmedian der Schweizer Bevölkerung $(41,6$ am 1. Januar 2011, Eurostat). Weiter hat gut ein Drittel der Befragten ein Hochschulstudium absolviert, was dem Schweizer Bevölkerungsschnitt entspricht (Bundesamt für Statistik Schweiz 2010). Damit stimmen die soziodemographischen Merkmale der befragten OnlineMediennutzer bis auf die Geschlechterdiskrepanz im Schnitt mit der Schweizer Gesamtbevö̈kerung überein.

Der typische Paid Content-Nutzer ist mit sehr hoher Wahrscheinlichkeit männlich, Anfang 40 und hat

Tab. 2: Häufigkeit von Paid Content-Käufen

\begin{tabular}{|l|l|}
\hline & Paid Content-Nutzer \\
\hline $\begin{array}{r}\text { Fallzahl } \\
\text { (Anteil) }\end{array}$ & $\begin{array}{l}\mathrm{n}=613 \\
(21,5 \%)\end{array}$ \\
\hline Erst einmal & $15,0 \%$ \\
\hline Gelegentlich & $63,8 \%$ \\
\hline Regelmäßig & $21,2 \%$ \\
\hline
\end{tabular}

(c) Medienwirtschaft

Wahrscheinlichkeit ein Hochschulstudium absolviert als ein Nichtnutzer. Der höhere Bildungsstand spiegelt sich auch im Einkommen wider. Etwa jeder dritte Paid Content-Nutzer hat nur jeder fünfte Nichtnutzer von Paid Content in diese Einkommensgruppe fällt. Paid Content-Nutzer unterscheiden sich daher mit ihren soziodemographischen Merkmalen deutlich von Nichtnutzern.

4.1.2 Nutzung der Paid Content-Angebote

Im Folgenden beleuchten wir die Häufigkeit der Nutzung, die Angebote sowie die Ausgaben für Paid Content Die

Abb. 1: Genutzte redaktionelle Paid Content-Angebote und andere Online-Bezahlangebote (Auswahl)

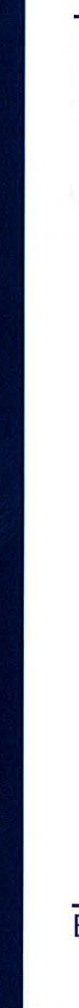

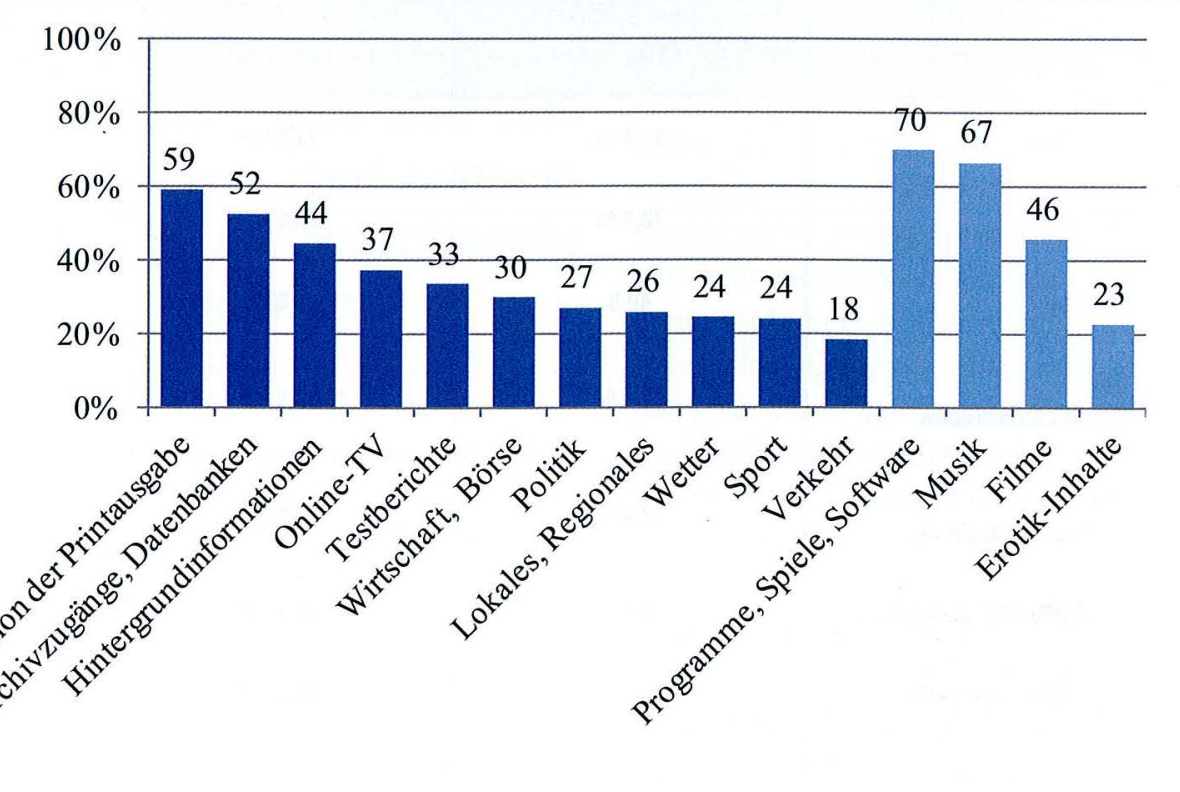

mPaid Content $\square$ Andere Online-Bezahlangebote ein Einkommen von über $8.000 \mathrm{CHF}$ (etwa 6.600 Euro), während

\section{Abb. 2: Ausgaben für Paid Content und andere Online-Bezahlangebote im letzten Jahr}

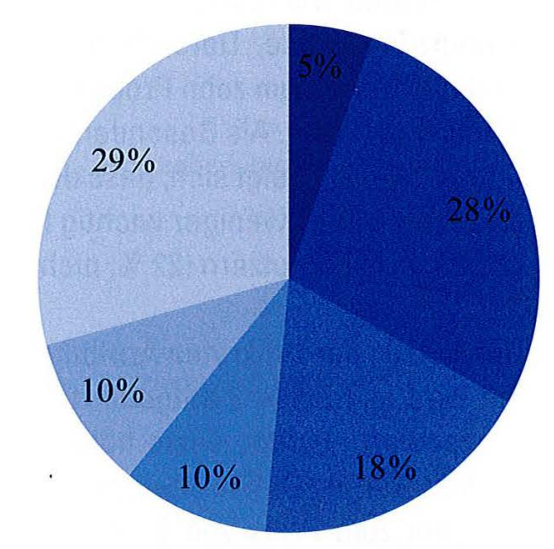

- Bis unter $10 \mathrm{CHF}$

- 10 bis unter $25 \mathrm{CHF}$

50 bis unter $75 \mathrm{CHF}$

175 bis unter $100 \mathrm{CHF}$

Mehr als $100 \mathrm{CHF}$
25 bis unter $50 \mathrm{CH}$ gentlich Online-Inhalte (ca. $82 \%$ ) (Tabelle 2). Jeder Fünfte kauft regelmäßig Paid Content-Angebote.

Die häufigsten Nutzungsformen von redaktionellen Paid Content-Angeboten sind das Lesen der elektronischen Version einer Printausgabe sowie der Zugang zu Archiven oder Datenbanken. Beides wurde von über der Hälfte der Paid Content-Nutzer bereits mindestens einmal gekauft (Abbildung 1). Darauf folgt der Kauf von Hintergrundinformationen an dritter Stelle. Online-TV und servic eorientierte Angebote wie Testberichte wurden von etwa einem Drittel der befragten Paid Content-Nutzer mindestens einmal gekauft. Spezifische Themen zu Wirtschafts- und Börseninformationen wurden von knapp einem Drittel bereits mindestens einm geturt. Angebote zu Politik bereits und Regional Wetter sowi Verkehr wurden jewils , dwa im Vergleich von den Wenigsten gekauft. Andere Online-
Bezahlangebote wie Programmdownloads, Spiele oder Software sowie Musik wurden dagegen von deutlich meh Paid Content-Nutzern gekauft als redaktionelle Angebote.

Abbildung 2 zeigt, dass die Ausgaben für Paid Content und andere Online-Bezahlangebote zwischen unter $10 \mathrm{CHF}$ (ca. 8,30 Euro) bis über $100 \mathrm{CHF}$ breit gestreut sind. Etwa die Hälfte der Nutzer hat im letzten Jahr über 50 CHF (ca. 40 Euro) für Bezahlangebote ausgegeben - drei von zehn Nutzern sogar über $100 \mathrm{CHF}$.

4.2 Welche Produkteigenschaften und Attribute von Paid Content-Angeboten führen zur höchsten Kaufwahrscheinlichkeit?

4.2.1 Online-Mediennutzer und Paid Content-Nutzer Diese Untersuchung erhebt mithilfe der ACA den Nutze von Paid Content-Angeboten. Dazu wird das Bündel „Paid

\section{Abb. 3: Verteilung der relativen Wichtigkeit der Produkteigenschaften von Paid Content}

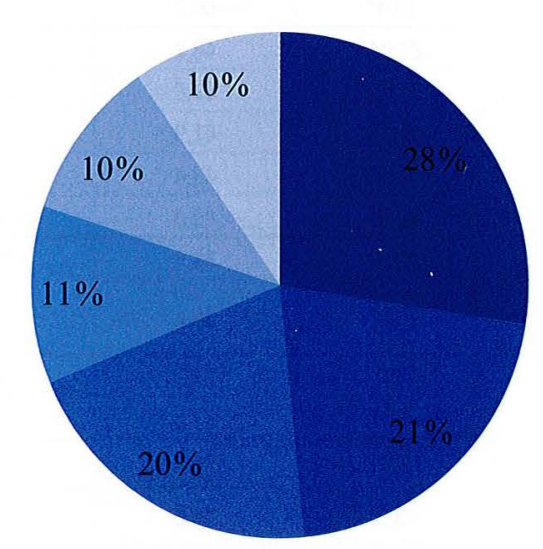

Preis

Produktinhalt

¿ahlungsabwicklung

Benutzungsweise

Abrechnungsmodell

Darstellung 
Content-Angebot" in die globalen Produkteigenschaften Preis, Produktinhalt, Zahlungsabwicklung, Benutzungsweise, Abrechnungsmodell und Darstellung zerlegt. Au einer zweiten Ebene werden diese globalen Produktegenschaften wiederum in konkrete Attribute untergliede (z. B. stationäre PC-Nutzung vs. mobile Nutzung für die Produkteigenschaft Benutzungsweise, s. zweite Spalte Tabelle 3). In die folgende Analyse werden alle befragten OnlineMediennutzer ( $n=2.850$ ) einbezogen. Anschließend werden Paid Content-Nichtnutzer $(n=2237)$ und Paid ContentNutzer $(n=613)$ verglichen.

Zunächst wird die Verteilung der relativen Wichtigkeit der jeweiligen ermittelt Je höher Prozent dieser auf die Kaufentscheidung. In Abbildung 3 stellen wir die relative Wichtigkeit der globalen Produkteigenschaften Preis, Produktinhalt, Zahlungsabwicklung, Benutzungsweise, Abrechnungsmodell und Darstellung für OnlineMediennutzer, also alle Befragten, dar. Der Preis $(28 \%)$ der Produktinhalt (21 \%) und die Zahlungsabwicklung (20
$\%)$ machen beinahe 70 Prozent der relativen Wichtigkeit

Tab. 3: Nutzen der Attribute von Produkteigenschaften von Paid Content-Angeboten

\begin{tabular}{|c|c|c|c|c|c|c|c|}
\hline \multirow[t]{4}{*}{$\begin{array}{l}\text { Produkt- } \\
\text { eigenschaft }\end{array}$} & \multirow[t]{4}{*}{ Attribut } & \multicolumn{2}{|c|}{ Online-Mediennutzer } & \multicolumn{2}{|c|}{$\begin{array}{l}\text { Paid Content- } \\
\text { Nichtnutzer }\end{array}$} & \multicolumn{2}{|c|}{ Paid Content- Nutzer } \\
\hline & & \multicolumn{2}{|c|}{$\begin{array}{l}\mathrm{n}=2.850 \\
(100 \%)\end{array}$} & \multicolumn{2}{|c|}{$\begin{array}{l}\mathrm{n}=2.237 \\
(78,5 \%)\end{array}$} & \multicolumn{2}{|c|}{$\begin{array}{l}\mathrm{n}=613 \\
(21,5 \%)\end{array}$} \\
\hline & & \multicolumn{2}{|c|}{ Nutzen } & \multicolumn{2}{|c|}{ Nutzen } & \multicolumn{2}{|c|}{ Nutzen } \\
\hline & & Mittelwert & $\begin{array}{c}\text { Standard- } \\
\text { abw. }\end{array}$ & Mittelwert & $\begin{array}{c}\text { Standard- } \\
\text { abw. }\end{array}$ & Mittelwert & $\begin{array}{c}\text { Standard- } \\
\text { abw. }\end{array}$ \\
\hline \multirow[t]{4}{*}{$\begin{array}{l}\text { Preis } \\
\end{array}$} & 2 CHF (ca. 1,70) & 78,9 & 61,3 & $83,8^{*}$ & 64,0 & $61,1^{*}$ & 46,2 \\
\hline & 5 CHF (ca. 4,10) & 27,4 & 27,4 & $28,8^{*}$ & 28,4 & $22,2^{*}$ & 22,8 \\
\hline & $10 \mathrm{CHF}$ (ca. 8,30) & $-26,1$ & 28,7 & $-28,0^{*}$ & 29,4 & $-19,2^{*}$ & 25,0 \\
\hline & $20 \mathrm{CHF}$ (ca. 16,60 ) & $-80,2$ & 60,3 & $-84,6^{*}$ & 63,2 & $-64,1^{*}$ & 44,7 \\
\hline \multirow[t]{5}{*}{ Produktinhalt } & $\begin{array}{l}\text { Aktuelle } \\
\text { Nachrichten }\end{array}$ & 26,2 & 42,4 & $28,1^{*}$ & 41,9 & $19,3^{*}$ & 43,3 \\
\hline & $\begin{array}{l}\text { Hintergrund- } \\
\text { informationen }\end{array}$ & 27,3 & 43,8 & $25,6^{*}$ & 43,0 & $33,2^{*}$ & 46,3 \\
\hline & $\begin{array}{l}\text { Archive/ } \\
\text { Datenbanken }\end{array}$ & $-0,9$ & 46,9 & $-4,3^{*}$ & 45,6 & $11,6^{*}$ & 49,7 \\
\hline & Wetter \& Verkehr & $-16,9$ & 47,9 & $-14,2^{*}$ & 46,6 & $-26,8^{*}$ & 50,9 \\
\hline & Börsenberichte & $-35,8$ & 51,2 & $-35,4^{*}$ & 50,2 & $-37,3^{*}$ & 54,6 \\
\hline \multirow{5}{*}{$\begin{array}{l}\text { Zahlungs- } \\
\text { abwicklung }\end{array}$} & Rechnung & 25,1 & 48,3 & $27,0^{*}$ & 48,2 & $18,1^{*}$ & 48,0 \\
\hline & Kreditkarte & 12,1 & 48,2 & $10,1^{*}$ & 47,8 & $19,4^{*}$ & 49,1 \\
\hline & Mobilfunkrechnung & 1,4 & 43,0 & $0,5^{*}$ & 41,9 & $4,7^{*}$ & 46,4 \\
\hline & Vorauszahlung & $-16,4$ & 43,8 & $-13,8^{*}$ & 43,4 & $-25,8^{*}$ & 44,2 \\
\hline & iTunes & $-22,2$ & 52,1 & $-23,8^{*}$ & 49,0 & $-16,5^{*}$ & 62,0 \\
\hline \multirow{2}{*}{$\begin{array}{l}\text { Benutzungs- } \\
\text { weise }\end{array}$} & Nutzung auf PC & 26,9 & 34,6 & 27,4 & 34,4 & 24,9 & 35,4 \\
\hline & $\begin{array}{l}\text { Nutzung auf Mobil- } \\
\text { telefon }\end{array}$ & $-26,9$ & 34,6 & $-27,4$ & 34,4 & $-24,9$ & 35,4 \\
\hline \multirow{2}{*}{$\begin{array}{l}\text { Abrechnungs- } \\
\text { modell }\end{array}$} & Abonnement & 8,1 & 37,5 & $7,3^{*}$ & 37,3 & $11,0^{*}$ & 38,1 \\
\hline & Nach Artikel & $-8,1$ & 37,5 & $-7,3^{*}$ & 37,3 & $-11,0^{*}$ & 38,1 \\
\hline \multirow[t]{2}{*}{ Darstellung } & Nur Text und Bild & 3,6 & 36,6 & 3.3 & 36,0 & 4,5 & 38,5 \\
\hline & Audiovisuell & $-3,6$ & 36,6 & $-3,3$ & 36,0 & $-4,5$ & 38,5 \\
\hline
\end{tabular}

beim Produktkauf aus. Diese drei Eigenschaften können damit als wichtigste Kaufentscheidungsfaktoren eingestuft werden. Die restlichen abgefragten Produktmerkmale (Benutzungsweise, Darstellung und Abrechnungsmodell) sind mit Werten um zehn Prozent bei der Kaufentscheidung weniger relevant. Als Besonderheit bei der Teilgruppe Paid Content-Nutzer zeigt sich, dass der Preis des Paid ContentAngebots etwas weniger wichtig für die Kaufentscheidung

Der Nutzen der konkreten Attribute der Produkteigenschaften wird in Tabelle 3 a fuefïht. Je positier ein nitlerer Nutzenwert ausfält, desto höher ist die Kaufwahrscheinlicheit Fïr die Online-Medionnutzer hat ein Paid ContentAngebot Zum Preis von 2 oder 5 CHF (ca. 1,70 b2 4,10 E E The (a) De Rechnung oder.Kreditkarte wird der Mobifunkrechnung, einer Vorauszahlung oder einer Zahlun über iTunes im Schnitt vorgezogen. Die Benutzungsweise

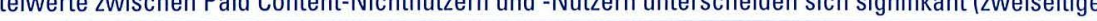

Abb. 4: Präferenzen zum Produktinhalt von drei Nutzertypen, Mittelwerte

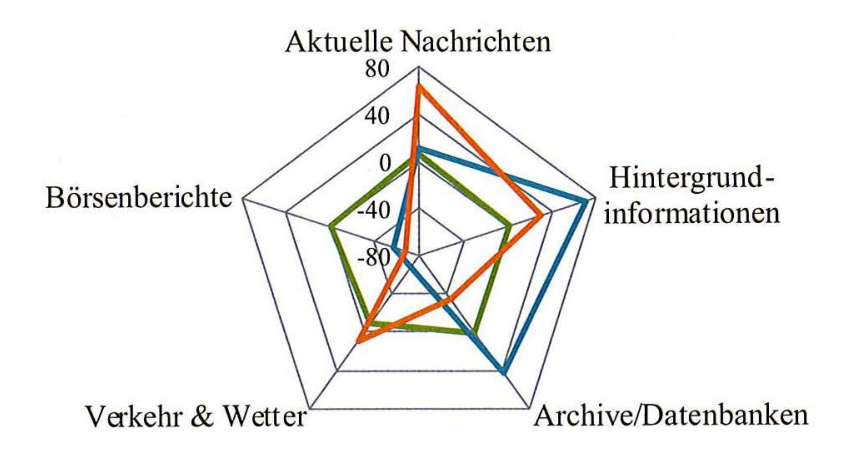

— Diversifizierer - Recherchierer - Aktuell Informierte

(C) Medienwirtschaft

auf dem PC hat insgesamt einen höheren Nutzen als die mobile Nutzung.

Unterschiede zwischen Paid Content-Nichtnutzern und Paid Content-Nutzern zeigen sich bei der Bewertung des Preises. Der Preis von 2 CHF wird von Nichtnutzern noch deutlicher bevorzugt als von Paid Content-Nutzern. Anhand der Standardabweichung bei Nichtnutzern läss sich ablesen, dass die Bewertung des Nutzens von $2 \mathrm{CHF}$ jedoch stark schwankt. Dies liegt v. a. daran, dass es besonders preissensitive Paid Content-Nichtnutzer gibt. Paid Content-Nutzer beurteilen Hintergrundinformationen am nützlichsten, während aktuelle Nachrichten für Nichtnutzer relevanter sind. Für Paid Content-Nutzer haben auch Archive und Datenbanken einen positiven Nutzen. Nichtnutzer bevorzugen eine Zahlung per Rechnung, während Nutzer die Zahlungsabwicklung per Rechnung und Kreditkarte gleich bewerten

Aus den Attributen in Tabelle 3 lässt sich nun ein Paid Con tent-Angebot mit dem vergleichsweise höchsten Nutzen bündeln: Die Kombination aus aktuellen Nachrichten und Hintergrundinformationen zu einem Preis von 2 oder $5 \mathrm{CHF}$ (ca. 1,70 bzw. 4,10 Euro), der per Rechnung oder Kreditkarte zahlbar ist, weist die jeweils höchsten Nutzenwerte und damit die höchste Kaufwahrscheinlichkeit auf.

\subsubsection{Paid Content-Nutzertypen}

Bisher haben wir hauptsächlich Online-Mediennutzer und Paid Content-Nutzer betrachtet. Nun sollten die Befragten in Nutzertypen unterteilt werden, um so die Kaufwahrscheinlichkeit für unterschiedliche Zielgruppen zu analysieren und Handlungsempfehlungen für Paid ContentAngebote geben zu können. Wir segmentieren dazu alle Befragten anhand ihrer Nutzenbewertung der jeweiligen Attribute der Produktinhalte (Aktuelle Nachrichten, Hintergrundinformationen, Archive/Datenbanken, Wetter \&

Abb. 5: Präferenzen zum Preis von drei Nutzertypen, Mittelwerte

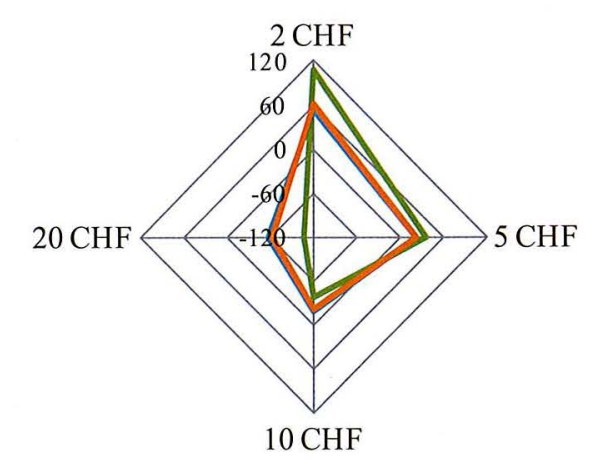

- Diversifizierer - Recherchierer -Aktuell Informierte 
Verkehr und Börsenberichte, s. zweite Spalte Tabelle 3). Mittels Cluster-Analyse können drei Gruppen gebildet werden, die sich in ihrer Nutzenzuschreibung von Produktinhalten unterscheiden4: Diversifizierer, Recherchierer und aktuell Informierte.

Für Diversifizierer hat kein Produktinhalt einen besonders stark ausgeprägten Nutzen (Abbildung 4). Insgesamt macht die Gruppe der Diversifizierer mit $43 \%(n=1.236)$ den größten Anteil der Befragten aus. Etwa jeder Fünfte dieses Clusters hat mindestens schon einmal redaktionelle Online-Inhalte gekauft $(n=235)$ - ist also Paid Content-Nutzer. Recherchierer finden Hintergrundinformationen und Datenbanken sehr nützlich (Abbildung 4) Knapp jeder Vierte aller Befrate nutzlich (Abbildung 4). Knapp jeder Vierte aller Befragte kann der Gruppe Rechrorerer zugewiesen werden $(24 \%$ $n=673$, von denen etwa jeder dnitte Nutzer von redaktionelem Paid Content ist lem Paid Content ist in dieser Gruppe am hochsten. Aktuel Informierte interessieren sich v. a. für allgemeine Inform tionen und zum Tell auch für Verkehr und Wetter sowie Hintergründe (Abbildung 4). Ein Drittel der Befragten sind aktuell
Informierte $(33 \%, n=942)$, wovon lediglich jeder sechste re Informierte $(33 \%, n=942)$, wovon lediglich jeder
daktionelle Paid Content-Erfahrung hat $(n=160)$

Auf der Grundlage von Hintergrundinformationen, aktuellen Nachrichten sowie Datenbankzugängen können vor allem für Recherchierer und aktuell Informierte konkrete Produktpakete erstellt werden. Beide Zielgruppen machen zusammen deutlich über die Hälfte (57\%) der Online-Mediennutzer aus. Wie es um die Zahlungsbereitschaft dieser Cluster in Detail beschaffen ist, wird im Folgenden analysiert.

4.3 Welche Rolle spielt der Preis?

Der Produktinhalt ist für Recherchierer und aktuell Informierte die relevanteste Produkteigenschaft, während der Preis ger Preis von 2 CHF ist besonders nützlich für Diversifiziere

(Abbildung 5). Recherchierer und aktuell Informierte würden 2 bis $5 \mathrm{CHF}$ aufwenden.

Vor allem Befragte, die keine Paid Content-Nutzer sind, beurteilen den geringen Preis von $2 \mathrm{CHF}$ deutlich nützliche (Tabelle 3). Über alle Cluster hinweg sehen Paid ContentErfahrene einen günstigen Preis nicht als per se besser an - aber Unerfahrene würden bei einem geringen Preis wahrscheinlicher Paid Content kaufen. Diese Differenz ist bei Diversifizierern mit Abstand am größten. Damit können Diversifizierer als preisaffin charakterisiert werden.

\section{Paid Content ist durchaus marktfähig.}

Die Zahlungsbereitschaft für Paid Content wurde in der vorliegenden Untersuchung neben der Preisabfrage in de vorlegenden Untersuchung neben der Preisabfrage in de Conjolt Analyse auch direkt erfragt. Wie vermutet, sind die Befragten grundsătzlich unwilig, fưr Online-Angebote zu zahlen. Die Zustimmung zur direkten Frage nach der Zahlungsbereitschaft für Online-Inhalte („, Ich finde es richtig, auch für Online-Angebote Geld zu bezahlen.") ist eher gering - wobei die Gruppe der Recherchierer etwas stärker zustimmt (Abbildung 6). Dies kann auf positive Erfahrungen mit Paid Content zurückgeführt werden, da vor allem die Paid Content-Nutzer unter den Recherchierern stärker zustimmen. Eine grundlegende Zahlungsbereitschaft fü Online-Inhalte besteht jedoch nicht. Auch im Freundeskreis der Befragten ist es nicht üblich, für Online-Inhalte Geld zu bezahlen. Vielmehr hat die Gratis-Mentalität weiter Bestand. Hat man aber bereits Erfahrung mit Paid Content dann wird eine Zahlung für Online-Inhalte zumindest weniger stark abgelehnt.

Ob das gleiche Angebot kostenlos auf einer anderen WebObe Untersuchung jedoch o

Abb. 6: Einstellung zur Zahlung für Paid Content-Angebote von drei Nutzertypen, Mittelwerte

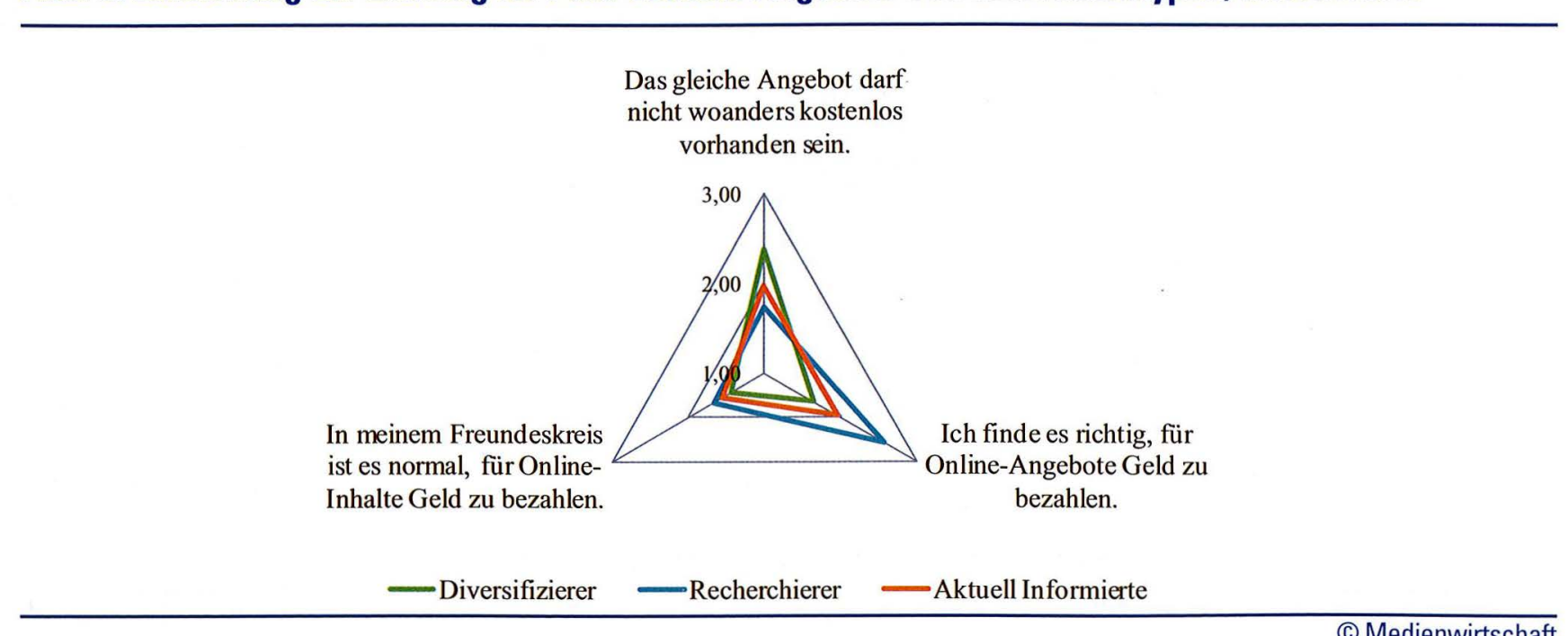

20 MedienWirtschaft 4/2012 erachtet. Einzig für die preissensiblen Diversifizierer ist diese Bedingung etwas wichtiger. Trotzdem widerspricht dieses Ergebnis der theoretischen Überlegung, wonach Paid Content nicht bei Free Content-Konkurrenz akzeptiert werden würde. Erklärung für das Ergebnis kann der Zusatzaufwand sein, vergleichbare Gratisangebote zu suchen. Wenn Paid Content schneller und einfacher aufzufinden ist als Free Content, dann verursacht der zusätzliche Suchaufwand für Free Content Kosten. Diese Suchkosten werden bei einem entsprechend tiefen Preis eines Paid Content-Angebots offenbar nicht in Kauf genommen, was die Ergebnisse eine Studie zum Online-Suchverhalten von Konsumenten bestätigt (Grewa/Lundsey-Mullikin 2006). Dies spiegeln auch die tigt (Grewal/Lundsey-Mullin 2006). Dies spiegeln auch die Angaben der Befres Kaufs wider. Paid Content-Nutzer hat das Angebot spontan interessient und sie wolten sich schnen in dargestellt). Daher war der Nuzzen der Geschwindigkeit beim Bezug der Informationen wichtiger als kostenlose Informationen. Weiter kann die Wahrgenommene Qualität des Angebots oder die Reputation der Medienmarke dazu führen, dass keine kostenlosen Angebote gesucht werden. Vor allem bekannte Medienmarken können davon profitieren. So kann ein Preis - wie weiter oben beschrieben - Qualität signalisieren (Pauwels/Weiss 2008) und eine Medienmarke über den funktionalen Nutzen hinaus symbolischen Nutzen stiften (Siegert 2001). Paid Content ist also durchaus markt fähig, wenn Free Content nur nach Zusatzaufwand zur Verfügung stünde. Damit müssen nicht-monetäre Kosten ebenfalls als Kaufkriterium für Paid Content betrachtet werden.

\section{5 Übertragbarkeit der Ergebnisse}

Einschränkend ist zu erwähnen, dass die Auswahl der Stichprobe, wie bei sämtlichen Online-Umfragen, nicht nach dem probe, wie beis af dor Gundage einer Grundgach dem Zulalspinz auf der Grict erfolgte. Damit ist die Stichprobe streng genommen nicht reprasentativ fir Deutsche Ein Konkr tenzieller Produkte ist daher nicht exakt abschatzbar. An dererseits gehen wir davon aus, dass die Ergebnisse zum einen autgrund der hohen Fallzanl und zum anderen durch das Aufschalten beifunf großen Medienunternehmen in de Deutschschweiz ein gutes Bild von Online-Mediennutzern zeichnen sollten. Dies zeigen auch die demographischen Merkmale, die mit früheren Studien und der Schweizer Bevölkerungsstatistik gut übereinstimmen. Weiter weist aus analytischer Perspektive die adaptive Conjoint Analyse (ACA) Schwächen auf, die sich aus der Durchführbarkeit de Erhebung ergeben. So muss man sich auf einige Produktmerkmale mit wenigen Ausprägungen beschränken, wobe die reale Kaufentscheidung komplexer vonstattengeht und von weiteren Faktoren abhängen kann. Beispielsweise wurde die Rolle der Medienmarke in der vorliegenden Untersuchung aus forschungsökonomischen Gründen vernachlässigt. Für Folgestudien wäre daher interessant zu prüfen inwiencit die Medienmarke eine Rolle bei der Beurteilung und Kaufentscheidung von Paid Content spielt.
Die Ergebnisse können weitestgehend auf Länder mit ähnlichen Bevölkerungs- und Medienstrukturen sowie ähn cher technologischer Entwicklung übertragen werden. Die Smartphone- und Tablet-PC-Verbreitung sind für die Zahl der potenziellen mobilen Nutzer von Paid Content ausschlaggebend. Wie weiter oben aufgezeigt, ist die Smartphone-Verbreitung in der Schweiz deutlich stärker vorangeschritten als in Deutschland. Eine Ursache kann im verfugbaren Haushaltseinkommen gesehen werden, das die Konsumausgaben determiniert. So lag die Höhe der Konsumausgaben im Durchschnitt je Haushalt und Monat in Deutschland 2009 bei etwa 2100 Euro (Statistisches Bundesamt 2011), während ein Schweizer Haushalt im Schnitt mit etwa 5.400 CHF (4.500 Euro) pro Monat im selben Jahr deutlich mehr ausgeben konnte (Bundesamt für Statistik BFS 2012). Haushaltsausaben für die Sparte Nachrichtenïbermittung in die auch Anschaffunsten für Mobiltelefone fallen, waten in der Schweiz CH bzw. 57 Euro in Dl. Ausgaben für Zei 列 (20 Eur in (26) 55. St baw. 7 Euro in D (Bundesamt fur Statistik BFS 2012. 35; Statistischos Bundesamt 2011. 550). Insgesamt sind zuming der Schweiz hoher als in Deutschland einzuschätzen. Dafür gibt es in Deutschland absolut gesehen mehr potenzielle Paid Content-Kaufer. Damit sehen wir vor allem bezüglich der Preisaffinität für Paid Content-Angebote Einschränkungen bei der Übertragbarkeit der Ergebnisse auf Deutschland sowie andere Länder mit geringeren Konsumausgaben. In der Schweiz bestehen für Paid Content besonders gute Voraussetzungen.

\section{Zusammenfassung und praktische}

\section{Schlussfolgerungen}

In dieser Untersuchung wurden 2.850 Online-Mediennutzer in der deutschsprachigen Schweiz im Dezember 2010 zu ihren Kaufentscheidungsfaktoren und Nutzungsgewohnheiten von Paid Content befragt. Das Nutzerprofil in der Stichprobe ist überwiegend männlich $(79 \%$. Wesentliche demographische Unterschiede zwischen Paid Content-Nutzern und -Nichtnutzern zeigen sich im deutlich höheren Bildungsabschluss und höheren Einkommen bei Nutzern. Zudem ist der typische Paid Content-Nutzer fast immer männlich.

Im Gegensatz zu der in der Literatur vorherrschenden Meinung gibt es durchaus selektiv Zahlungsbereitschaft für online angebotene redaktionelle Inhalte. Aus unserer Sicht ist daher die generelle These zur geringen Zahlungsbereitschaft zu relativieren. Obwohl die allgemeine Zahlungsbereitschaft für Online-Angebote gering ist, zeigt die Conjoint Analyse, dass für spezifische Inhalte ein Preis verlangt werden kann. Die Resultate lassen insgesamt den Schluss zu, dass vor allem der kostenpflichtige Inhalt selbst, sein Preis und das Z ahlungssystem die Kaufentscheidung determinie- 
ren. Zudem konnte die Bedeutung der Exklusivität von Paid Content-Angeboten spezifiziert werden. Wenn Paid ContentInhalte auf Interesse stoßen, schnell konsumiert werden wollen und ein Zugang zu Free Content mit Suchaufwand verbunden ist, kann Paid Content attraktiver wahrgenommen werden

Vor allem der kostenpflichtige Inhalt selbst, sein Preis und das Zahlungssystem determinieren die Kaufentscheidung.

Als zahlungsbereite, Paid Content-affine Nutzergruppen können vor allem Recherchierer und in geringerem Ausmaß aknen vor allem Recherchierer und in geringerem Ausmaß ak-
tuell Informierte zusammengefasst werden, die zusammen $57 \%$ aller befragten Online-Mediennutzer ausmachen und spezifische Informationsbedürfnisse aufweisen. Ein ideales Produkt für diese beiden Nutzertypen würde die folgende Eigenschaften als Bündel aufweisen:

- Hintergrundinformationen und Reportagen

- Zugang zu Datenbanken und Archiven

- Aktuelle Informationen

- Bequeme Kaufabwicklung per Rechnung oder Kreditkarte

- Kosten zwischen 2 und maximal 5 CHF pro Abrechnungsoder Nutzungseinheit (abhängig vom Produktinhalt). Fuir Neukundengewinnung ist ein Preis von 2 CHF empfehlenswert, do er einen höheren Nutzen für Personen aufuest die noch keine Paid Content-Erfahrung gemacht haben.

Insgesamt schließen wir aus der Untersuchung, dass Pomedien, sonden auch Onlin-Medien werden aurgund vo Hintergu dinfurn a handen in gern vestiche lis ger verglechswese einfach befriedige werden, da sie sich auf das journalistische Kerngeschâft beziehen. Online-Nutz werden zwar aller Voraussicht nach keine höhere Zahlungsbereitschaft fưr publizistische Online-Medieninhalte ausbilden als für die "Top-3-Online-Seller" Software, Games und Musik - aber mit konkreten bedürnnisorientierten Angeboten können auch Verlage zukünftig ein gutes Stück Online-Umsatz für Paid Content abbekommen.
Anmerkungen

1 Auch mit dem dritten am Markt zu beobachteten Grundmodell für Pay das Wall Street Journal positive Effahrungen gemacht und verzeichneis keine signifikanten Nutzerrückgänge

2 Im Folgenden wird das psychologische Kaufentscheidungskriterium

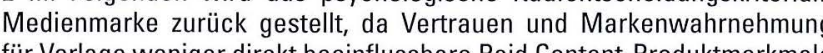
darstellen Nichtdestotrotz sind diese wichtig und sollten weiter erforsch

Online-Medien (Unique Clients im Dezember 2010): 20 min.ch (2,2 Mio. blick.ch (2 Mio.), nzz.ch (1,3 Mio.), Newsnetz-Portal (1,8 Mio.) und suedost4 Der Silhouetten-Kohäsions- und Trennungswert ergibt eine Clusterab-
grenzung von 0,5 , was eine mittlere Grö̉e darsteltt. Dies weist auf eine
ausreichende interne Homogenität und externe Heterogenität der einzelausreichende inter
nen Gruppen hin.

Literatur

BBC (2010): Times and Sunday Times readership falls after paywall (http:/

BTKOM (2010): Wachsende Zahlungsbereitschaft für Qualitätsjiourna01.10 .2012 .

(2012): Smartphone-Funktionen: Internet wichtiger als TelefoNieren (http://Www.bitkom.org/files/documents/BITKOM_Presseinfo

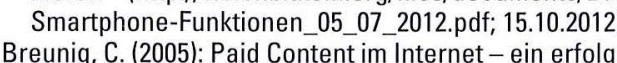

Breunig, C.C. (2005): Paid Content
modell? Marktchancen kostenpflichtiger - ein erfolgreiches Geschäfts pektiven (2005), H. 8, S. 407-418.
B. Buchholz, A./Sandhu, S./Kupczzk, T, T/Zerfaß, A. A. (2004): Anticipating Con-
tent Technology Needs. Final Report. (hittp://wwww.communicationma tent Technology Needs. Final Report. (http://www.communicationma
nagement.de/fileadmin/cmgt/PDF_Publikationen_download/ACTeN FINAL_REPORT.pdf

Bundesamt tür Statistik BFS (2012): Haushaltsbudgeterhebung 2009. Kom tal/de/index/news/publikationen.h.hm!? publicationlD=4825; 01.10.2012.

Bundesamt für Statistik Schweiz (2010): Szenarien für das Bildungssystem - Analysen. Szenarien 2010-2060 zum Bildungsniveau der Bevö̈lkerung.

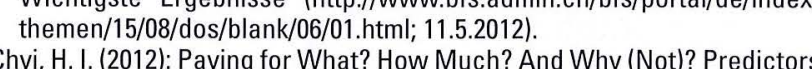
Chyi, H. I. . (2012): Paying for What? How Much? And Why (Not)? Predictors
of Paying Intent for Multiplatform Newspapers. In: International Jourof Paving Intent for Multiplattorm Newspapers. In: Interna
nal on Media Management, 14. Jg. (2012), H. 3, S. 227-250.

Cook, J.E./Shahzeen, A.Z. (2012): Paving for what was free: Lessons from the New York Times Paywall. In: Cyberpsychology, Behavior, and Social Networking, 15. Jg. (2012), H. 12, S. 1-6.

政 h. S. (2005): Paid Content und Paid Service bei einer Online-Redakion (am Ben) net: Deutschland 2012

( frame on the effects of price range and the number of competitors on Science, 34. Jg. (2006) H. 1.S. 55-62.

all, E. (2010): Lessons for U.S. media from European paid-content plays. In: Advertising Age, 81. Jg. (2010), H. 9, S. 10

2009): Consumer selection of e-commerce websites in a B2C environment: A discrete decision choice model. In
IEEE Transactions on Systems: Mas

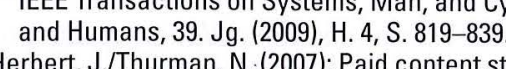

An empirical study of British news An empirical study of Britith newspapers' online
Journalism Practice, 1. I. g. (2007), H. 2, S. 2. 208-22

Hogenkamp, P./Baumeler, R./Küffer, M. (2011): NZZ setzt auf Konverge

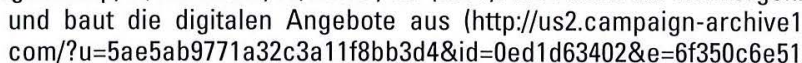
com/? 11.10 .2012

Hutter, T. (2012): Paid Content als Erfolgsmodell? Eine Untersuchung der brücken 2012

Krim, H.W.W.Xu, Y./Gupta, S. (2012): Which is more important in Interne shopping, perceived price or trust? In: Electronic Commerce Researc . . (2012): KPN Jg. (2012), H. 3, S. 241
Mitchell, A./Rosenstiel, T./Houston Santhanam, L./Christian, L. (2012): Fu-
ture of mobile news. The explosion in mobile audiences and a close look at what it means for news 2012

Mogerle, U. (2009): Substitution oder Komplementarität? Die Nutzung vo files/files/NET-Metrix-Mobile/Mobile-Report/Factsheet/NMM Mobile-

Report Factsheet.pdf; 27.9.2012).
NET-Metrix AG: NET-Metrix-Audit: Online-Nutzungsdaten Dezember 2010
(ST) 15.10.2012)

Nitschke, T./Sattler, H. (2005): Präferenzstrukturen und Zaahlungsberei-

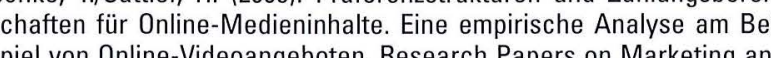
spiel von Online-Videoangeboten. Research Papers on Marketing and
Retailing Band 26 (http://www.universitatit-hamburg.de/fachbereicheeirrichtungen/fbo3/hm//RP26.pdf; 11.5 .52012 .

D/Gwinner, K. P./Wiertz, C. (2009) Toward a theory of repeat purchase drivers for consumer services. In
Journal of the Academy of Marketing Science, 37. Jg. (2009) H. 2, S. Science, 37. Jg. (2009), H. 2, S. Pauwels, K..Neiss, A. (2008): Moving from Free to Fee: How Online Firms Marketing, 72. Jg. (2008), H. 3, S. 14-31.

Rademacher, P. (2007): "Content is King - aber wer zahlt dafür?". Die Bekeutung von Kaufpreis und Micropaymentsystemen für den OnlineveSchweiger, W. (Hrsg.), Neue Technik, neue Medien, neue Geselllschaft.
Ökonomische Herausforderungen der Onlinekommunikation, München Okonomische Heraustorderungen der Onlinekommunikation, München,
S. 69-96.

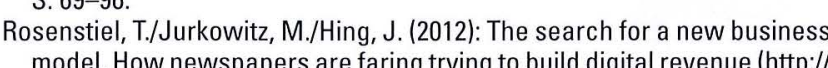
model. How newspapers are faring trying to build digital revenue (http:///
www.journalism.org/analysis_report/search_new_business_model www.journ. publizistischer Qualität und ökonomischem Erfolg? In: Fabris, H. H. Rest, F. Hrrsg.l, Qualitata als Gewinn. Salzburger Beitrage zur Qualitäts-

Stanl, F. (2005): Paid Content. Strategien Zur Preisgestaltung beim elektro-
nischen Handel mit digitalen Inhalten. Zugleich Dissertation an der HSS N. Gallen, Wiesbaden 2005 .
St

: Statistisches Jahrbuch für die BundesreThe New York Times Company (2012): The New York Times company reports 2012 second-quarter results (httr: $: / /$ /phx.corporate-irinet 11.10.2012).

The Nielsen Company (2010): Changing models. A global perspective on paying for content online http://blog

Wang, C. L./Ye, L. R./Zhang, Y./Nguyen, D.-D. (2005): Subscription to fee based online services: What makes consumer pay for online content?

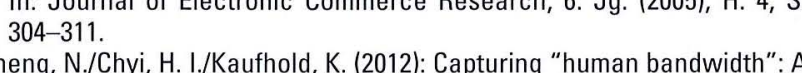
Zheng, N./Chyi, H. I./Kauthold, K. (2012): Capturing human bandwidth": A
multidimensional model for measuring attention on web sites. In: Inter- 